Casos Clínicos

Arch. Esp. Urol., 61, 6 (744-748), 2008

\section{PECOMA PÉLVICO DEL LIGAMENTO ANCHO CON METÁSTASIS GANGLIONARES: PRESENTACIÓN DE UN CASO Y REVISIÓN DE LA LITERATURA}

Raul Tobalina Bonis, Tomás Pérez Domínguez, Fernanda Relea Calatayud, Enrique Cazenave Jiménez, M. A. Piris y Nemesio Jiménez LópezLucendo.

Servicios de Cirugía General, de Anatomía Patológica y Urología del Hospital Gutiérrez Ortega y CNIO.

Valdepeñas. Ciudad Real. España.

Resumen.- OBJETIVOS: Describimos y documentamos el primer caso de PEComa publicado en España según la base de datos Pub Med. Y revisamos la bibliografía existente sobre este tipo de tumores.

MÉTODOS/RESULTADOS: Se Trata de una paciente de 39 años con un tumor pélvico de $9 \mathrm{cms}$. descubierto en

Raúl Tobalina Bonis Servicio de Cirugía Hospital Gutiérrez Ortega Avda. Estudiantes, s/n. 13300 Valdepeñas. Ciudad Real. (España). raulymer@terra.es

Trabajo recibido: 6 de noviembre 2007 una revisión ginecológica rutinaria. El TAC muestra múltiples adenopatías periaórticas que se extienden hasta vena renal izquierda El diagnóstico de PEComa se obtiene por punción con aguja gruesa. Se realiza exéresis del tumor junto a anexo izquierdo y linfadenectomía aorto-iliaca. El resultado patológico confirma el diagnóstico tras la inmunohistoquímica con actina de músculo liso y HMB-45. No recibió tratamiento adyuvante. Tras un año de seguimiento la paciente no muestra en el TAC signos de recidiva. Se realiza una amplia búsqueda bibliográfica en Pub Med encontrando 73 referencias a este tipo de tumor cuyas conclusiones se exponen en este articulo.

CONCLUSIONES: El tumor de celulas epitelioides perivasculares, PEComa, es una neoplasia mesenquimal de muy baja incidencia y con malignidad incierta. Tumores muy raros: el angiomiolipoma epitelioide, la linfangioleiomiomatosis, el linfangiomioma, el tumor miomelanocitico del ligamento falciforme, el tumor pulmonar de celulas claras "de azucar" y su variante extrapulmonar; son antiguas descripciones de lo que ahora es una única entidad tumoral denominada PEComa. Aun no de ha descrito la célula que en tejidos normales da origen a este tumor. Afectando principalmente a mujeres en edad media, pueden encontrarse en cualquier lugar anatómico. Su tratamiento es quirúrgico aunque se desconoce el papel de la linfadenectomía y del tratamiento adyuvantes.

Palabras clave: PEComa. Célula epitelioide perivascular. Ligamento ancho. Sarcoma. Linfangiomiomatosis. HMB-45.

Summary.- OBJECTIVE: We describe and document the first case of PEcoma published in Spain following the PubMed database. We review the bibliography about these tumors.

METHODS/RESULTS: 39-year-old female patient with a 9 $\mathrm{cm}$ pelvic tumor discovered in a routine gynecologic review. CT scan showed multiple periaortic adenopathies extending up to the left renal vein. The diagnosis of PEcoma was obtained by needle biopsy. Tumor excision including left annex and aortoiliac lymphadenectomy were performed. Pathology confirmed the diagnosis after immunohistochemical study with smooth muscle actin and HMB-45. No adjuvant treatment was given. After one year of follow-up the patient does not show signs of recurrence of the CT scan. A PubMed search was performed finding 73 references about this kind of tumor the conclusions of which are exposed in this article.

CONCLUSIONS: Perivascular epithelioid cell tumor, PECo$m a$, is a very low incidence mesenchymal neoplasia with uncertain malignancy. Very rare tumors such as epithelioid angiomyolipoma, lymphangioleiomyomatosis, lymphangio- 
myoma, myelomelanocytic tumor of the falciform ligament, "sugar" clear cell lung tumor and its extrapulmonary variant are all descriptions of what is now a unique tumoral entity named PEcoma. It has not been described yet the normal tissue cell giving origin to this tumor. It affects mainly middle age women, and may appear in any anatomical site. Treatment is surgical, although the role of lymphadenectomy and adjuvant treatment is unknown.

Keywords: PEcoma. Perivascular epithelioid cell. Broad ligament. Sarcoma. Lymphangiomatosis. HMB-45

\section{INTRODUCCIÓN}

El PEComa, tumor de células epitelioides perivasculares, de reciente descripción, agrupa una familia de tumores mesenquimales que comparten la característica de expresar simultáneamente marcadores inmunohistoquímicos melanocíticos y de músculo liso. Aunque habitualmente son benignos, pueden metastatizar por vía hemática y linfática. Hay casos descritos en cualquier edad (3-87 años) con una media de 40 años y afectan a mujeres en una proporción de 7:1 que se mantiene en 4:1 excluyendo el origen ginecológico que es el más frecuente. Un pequeño grupo, el PEComa del ligamento falciforme, se encuentra sobre todo en niños. Su localización anatómica es muy variable aunque predomina el origen ginecológico $30-35 \%$, vísceras $10 \%$ y el resto están descritos en partes blandas y piel por todas las regiones anatómicas, también en hueso. Hay 5 casos descritos en los que el PEComa estaba asociado a la esclerosis tuberosa. Su tratamiento consiste en la extirpación quirúrgica, apenas hay casos de tratamientos quimio o radioterápicos adyuvantes.

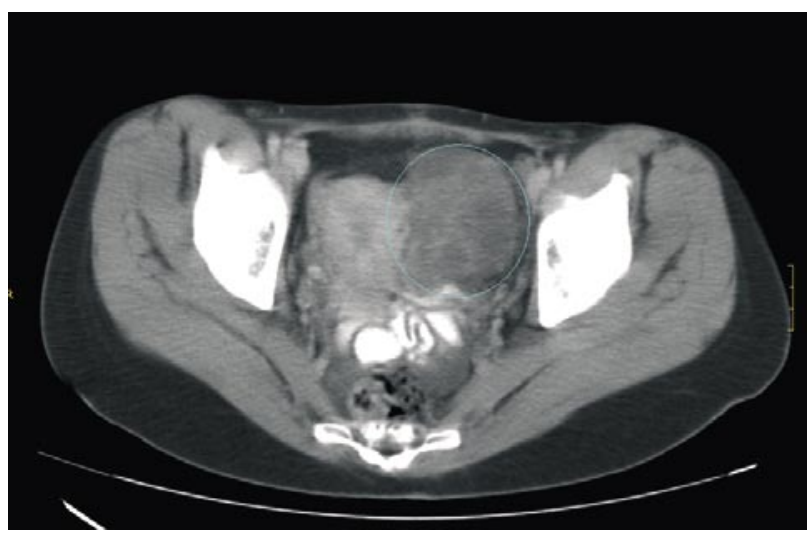

FIGURA 1. Lesión primaria en anejo izquierdo de carácter sólido-quístico.
Hasta la publicación de Folpe y cols. en diciembre de 2005 se habían descrito 61 casos en la literatura de habla inglesa a los que añadió 26 casos más.

\section{CASO CLÍNICO}

Mujer de 39 años que en una revisión ginecológica se aprecia masa en anejo izquierdo. Presenta elevación del Ca 19-9 de 192.6 con alfafetoproteina, CEA y Ca 125 en valores normales. Los estudios de imagen, ecografía, TAC (Figuras 1 y 2) y RNM muestran una masa pélvica sólido quística de $9 \times 6 \times 3 \mathrm{cms}$ anexial izquierda así como múltiples adenopatías peri-iliacas y periaorto-cava que se extienden cefálicamente hasta venas renales. La urografía y el enema opaco no muestran alteraciones.

Se realiza laparoscopia ginecológica diagnóstica que muestra que dicha masa se localiza en ligamento ancho uterino izquierdo sin afectación uterina, ni de trompa y ovario izquierdos, asi como tampoco infiltración peritoneal siendo la paciente derivada al Servicio de Cirugía General. Con la sospecha de linfoma retroperitoneal se realiza BAG (biopsia con aguja gruesa) en la que sé diagnosticó un tumor de células epitelioides perivasculares (PEComa). Se programa la cirugía en la que se realiza extirpación de la masa pélvica incluyendo anejo izquierdo así como linfadenectomía de vasos iliacos izquierdos y periaortocava hasta vena renal.

El estudio macroscópico de la pieza quirúrgica muestra una lesión bien definida, de coloración rosada, consistencia elástica y aspecto esponjoso (Figura 3), con pequeños quistes y abundante material hemático en su interior.

El estudio microscópico (Figuras 4 y 5) muestra un entrelazado de canales y pequeños quistes en cuyas paredes se observan células de aspecto epitelioide con

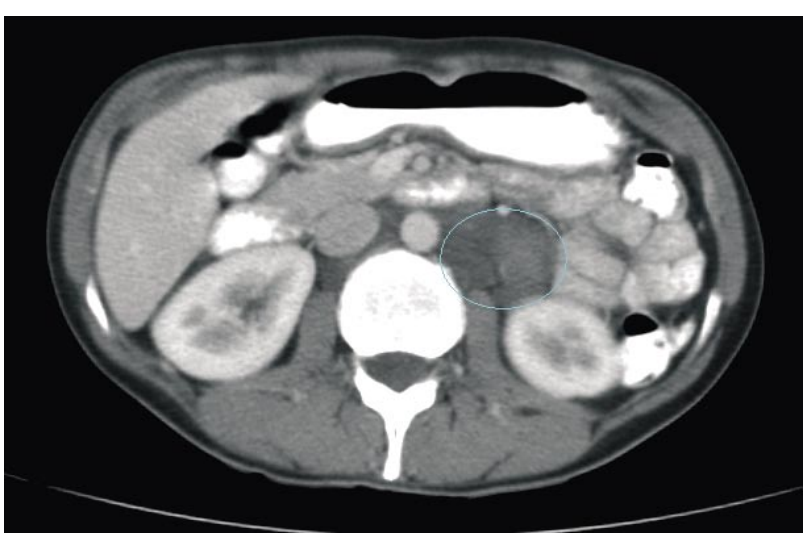

FIGURA 2. Adenopatias regionales que se extienden junto a la aorta hasta venas renales. 


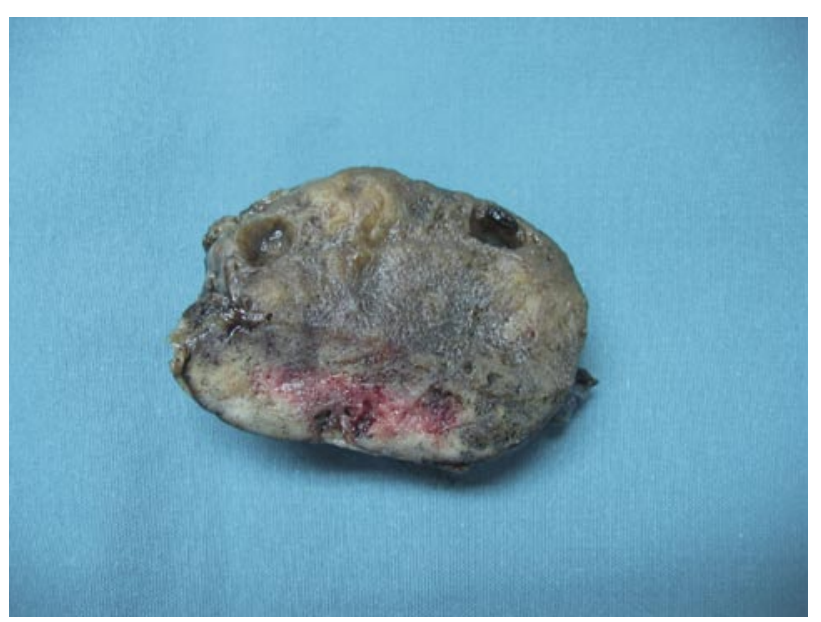

FIGURA 3. Imagen macroscópica de la tumoración. Se trata de una lesión sólida bien definida, de aspecto esponjoso, coloración rosada-blanquecina y con pequeños quistes en su interior.

abundante citoplasma que puede ser claro o finamente granular, con moderado pleomorfismo celular y escaso numero de mitosis.

El estudio histoquímico del tumor (Figuras 6 y 7 ) muestra positividad para actina muscular específica, actina de músculo liso y desmina, y positividad focal para el marcador melánico HMB-45. El índice proliferativo Ki67 es bajo.

La neoplasia fue diagnosticada de PEComa del ligamento ancho, con metástasis en los 19 ganglios regionales aislados.

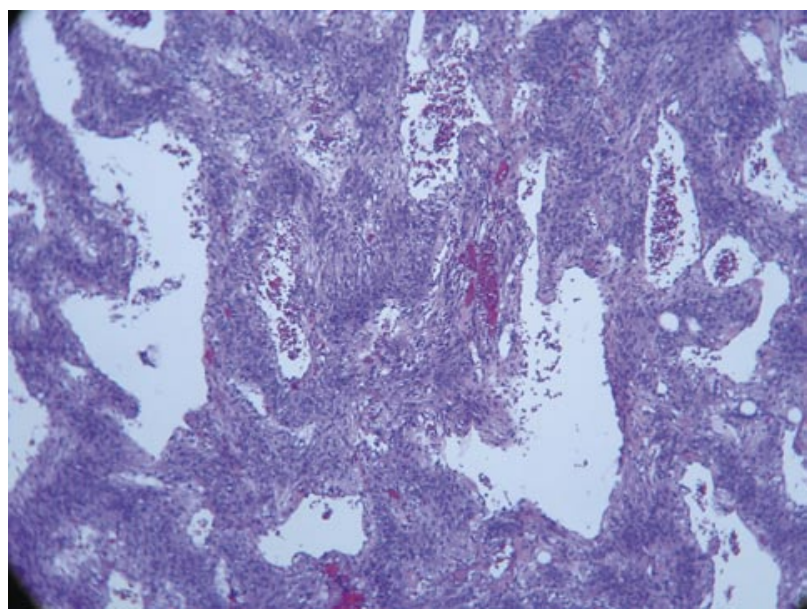

FIGURA 4. Imagen microscópica de la tumoración compuesta por canales vasculares en cuyas paredes se observa una proliferación de células epitelioides com moderado pleomerfismo $(H E, 10 x)$
Tras 12 meses de seguimiento, la paciente permanece asintomática y sin signos radiológicos de recidiva ni nuevas adenopatías.

No ha recibido tratamiento quimioterápico, ni radioterapia adyuvantes.

\section{Discusión y revisión de la literatura}

El PEComa, tumor de células epitelioides perivasculares, es un tumor de origen mesenquimal. Este término redefine a un grupo de neoplasias que inicialmente fueron descritas independientemente y que han demostrado tener un origen común.

Esta denominación de PEComa incluye:

- El angiomiolipoma descrito en 1900 y su forma epitelioide de comportamiento más agresivo.

- La linfangioleiomiomatosis en la década de los 70.

- El tumor pulmonar de células claras "de azucar" posteriormente también descrito en el páncreas e hígado.

- El linfangiomioma.

- Y el tumor miomelanocítico de células claras del ligamento falciforme.

La asociación entre el angiomiolipoma, la linfangioleiomiomatosis y el tumor pulmonar de células claras se ha constatado gracias a que comparten el marcador inmunihistoquimico HMB-45. También se ha descrito un caso de asociacion de la linfangioleiomiomatosis y el tumor de células claras "de azucar"en un mismo paciente con esclerosis tuberosa y un caso de tumor híbrido entre ambos.

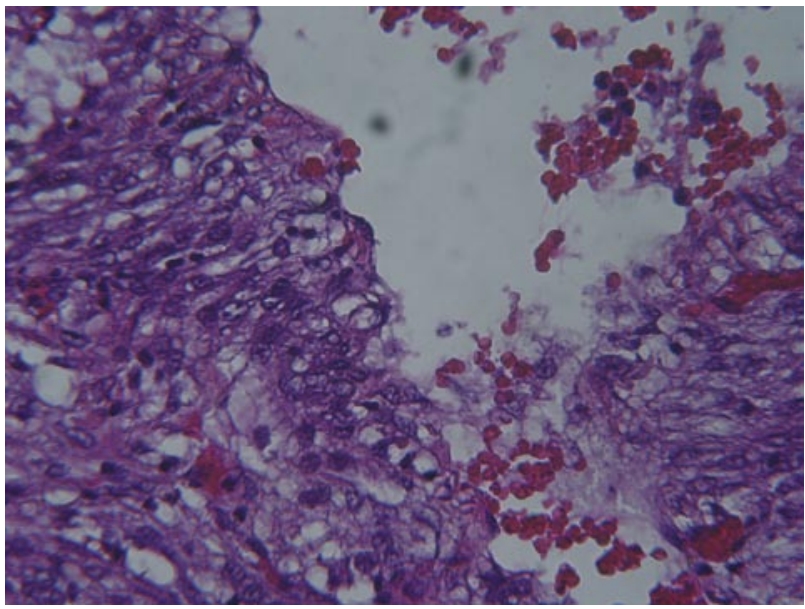

FIGURA 5. Imagen a mayor aumento de la celularidad tumoral. Son células epitelioides de citoplasma eosinófilo, ocasionalmente vacuolados y núcleos con moderado pleomorfismo. (HE, 40x) 
La primera descripción data de 1992 por Bonetti, el termino PEComa no se acuñó hasta 1996 por Zamboni y en 2002 fue reconocido por la OMS.

Desde los inicios se ha asociado el PEComa con la esclerosis tuberosa, pero la inmensa mayoría de los casos son esporádicos.

La célula originaria de estos tumores, la célula epitelioide perivascular, no ha sido descrita en tejidos normales sanos. La localización perivascular y la positividad de la actina sugieren un origen en el pericito que no ha sido demostrado.

Tampoco se ha descrito una lesión precursora de estos tumores, salvo la descripción de Weinreb de células claras tumorales adyacentes a la lamina basal luminal de los capilares que podrían estar en relación con lesiones precursoras. Han sugerido el termino PECosis para describir esta entidad histológica.

Los PEComas se caracterizan por una morfología epitelioide o fusocelular, cuyo citoplasma va desde claro a eosinofilo, de localización perivascular y que coexpresan marcadores melanociticos HBM-45 y de músculo liso preferentemente la actina.

En un estudio retrospectivo de sarcomas de partes blandas encontraron en 31 casos consecutivos 3 PEComas en tumores previamente catalogados como sarcomas de alto grado indiferenciados. Por lo que proponen descartar el PEComa en sarcomas de difícil clasificacion.

Hasta la fecha se han publicado sobre todo casos aislados, aunque hay algunas series de casos: siete casos de pecoma del ligamento falciforme en niños y jóvenes, una serie de 8 casos en utero, otra de 4 casos abdominopelvicos, otra de 4 casos de tumor de células claras

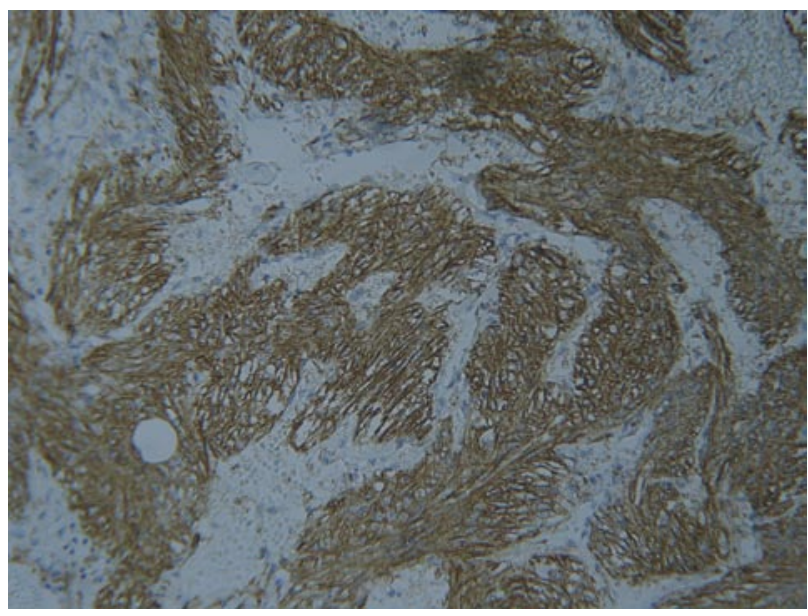

FIGURA 6. Marcaje inmunohistoquímico difuso de las células tumorales con actina de músculo liso. de "azucar" extrapulmonar y de PEComa cutáneo con 7 casos.

Pero la mayor serie, la presentada por Folpe y cols. con 26 casos, añade la revisión de todos los casos publicados hasta 2005 en la literatura de habla inglesa. En esta revisión se intenta establecer qué características tumorales permiten predecir el comportamiento biológico del PEComa como benigno, indeterminado o maligno.

La localización de los PEComas es muy diversa predominando la uterina. Recientemente en una revisión retrospectiva de 25 leiomiosarcomas uterinos se ha encontrado que el $36 \%$ de ellos expresan marcadores melanociticos (HMB-45) característicos del PEComa. Los autores proponen que no se utilice el termino PEComa para describir a los leiomiosarcomas uterinos incluidos los leiomiosarcomas de células claras y los que expresan reactividad de HMB-45. Se han encontrado también casos en cervix y vagina.

Se han descrito 4 casos en ligamento ancho uterino en este artículo describimos el quinto.

Se han descrito PEComas en partes blandas de los miembros, retroperitoneo, pelvis y ligamento falciforme. Tambien afectan el tracto urinario, riñón, vejiga, y próstata, en tubo digestivo (desde duodeno a recto) donde plantean diagnostico diferencial con los GIST, higado, coledoco y páncreas. Tambien se han encontrado en órbita, piel, mama, inguinal, mucosa oral, peritoneo, pared abdominal, aurícula izquierda y 2 casos en hueso (tibia y base del cráneo).

Los PEComas típicamente se diagnostican cuando alcanzan un gran tamaño, lo que refleja la falta de síntomas

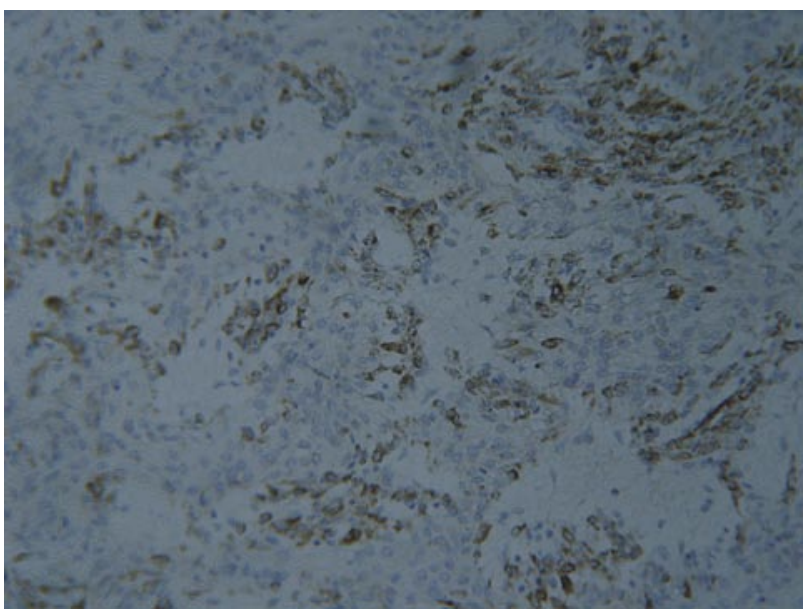

FIGURA 7. Marcaje inmunohistoquímico focal en algunas células tumorales con HMB-45. 
durante su crecimiento. El tamaño medio al diagnostico es de $5 \mathrm{cms}$.

Salvo que se plantee el diagnostico diferencial con el linfoma, como es el caso que presentamos debido a las múltiples adenopatías regionales, se debe ir a cirugía evitando biopsiarlo, del mismo modo que sucede en los sarcomas, para evitar su diseminación.

Su comportamiento biológico es variable. La mayoría son benignos, pero se han reportado metástasis hasta 9 años después de extirpar el tumor lo que aconseja realizar un seguimiento a largo plazo para los casos benignos.

Los casos catalogados de malignos se deben a la presencia de metástasis ganglionares o a distancia en el momento del diagnóstico o a lo largo del seguimiento. Los angiomiolipomas renales típicos a pesar de poder ser multifocales y tener metástasis ganglionares se califican como benignos; no sucede así con los angiomiolipomas epitelioides cuyo comportamiento sí puede ser maligno.

Evaluar la malignidad en un tumor no metastásico es complejo ya que no se han descrito rasgos histológicos que permitan con seguridad predecir su comportamiento, si bien algunos autores han propuesto considerar el tamaño $>5 \mathrm{cms}$, la actividd mitotica ( $\geq 1 / 50 \mathrm{HPFs}$ ) y la necrosis como principales marcadores de agresividad y proponen considerar también la invasión linfovascular, la alta celularidad y el alto grado nuclear (polimorfismo, células gigantes multinucleadas) como marcadores de malignidad de menor entidad que los anteriores.

El tratamiento de los PEComas consiste al igual que los sarcomas, en la escisión quirúrgica con márgenes adecuados. Se desconoce el papel de la linfadenectomía regional en los PEComas sin metastasis ganglionares evidentes. Hay casos publicados de tratamiento quirúrgico de las recidivas y las metástasis.

El papel de la adyuvancia no esta establecido y prácticamente solo se utiliza la quimioterapia y la radioterapia para tratamiento paliativo en PEComas metastásicos y con resultados dispares. En algunas publicaciones orientadas a la anatomía patológica no se especifica que quimioterapia ni que radioterapia fue administrada a los pacientes.

Se ha utilizado inmunoterapia adyuvante con Interferon alfa $2 b$, que aporta efecto anti angiogenico, en un paciente de 48 años con pecoma de $3 \mathrm{cms}$ en la vejiga fistulizado a intestino delgado. A los 48 meses el paciente esta libre de enfermedad

Se describe la asociación, en una paciente de 9 años, de dos ciclos de vincristina, ifosfamida y doxorrubicina preoperatorios en un tumor uterino con adenopatías regionales (consiguiendo una reducción del 10\% del ta- maño tumoral) y radioterapia postoperatoria mas otros 6 ciclos. Notifican éxito de este régimen a los 18 meses de seguimiento.

Dado el efecto del Imatinib en el tratamiento de los GIST, se ha buscado el marcador CD 117 en los PEComas encontrándose dicho marcador sólo en casos aislados.

El única ocasión en que se ha descrito la utilización de imatinib en un paciente con pecoma renal no se encontró ninguna respuesta en el tumor, que previamente habia sido tratado con dacarbacina, y vincristina.

Debido a que el PEComa es un fumor de muy baja incidencia no es posible establecer claros criterios para su manejo clínico, tratamiento adyuvante y el seguimiento debe hacerse a largo plazo incluso el aquellos considerados como benignos.

\section{BIBLIOGRAFÍA Y LECTURAS RECOMENDADAS (*lectura de interés $y^{* *}$ lectura fundamental)}

*1. SIMPSON, K.W.; ALBORES-SAAVEDRA, J.: "HMB45 Reactivity in Conventional Uterine Leiomyosarcomas". Am. J. Surg. Pathol., 31: 95, 2007.

2. KIM, H.J.; LIM, S.J.; CHOI, H. y cols.: "Malignant clear-cell myomelanocytic tumor of broad ligament: A case report". Virchows Arch., 448: 867, 2006.

**3. HORNICK, J.L.; FLETCHER, C.D.: "PEComa: What do we know so far?". Histopathology, 48: 75, 2006.

4. FINK, D.; MARSDEN, D.E.; EDWARDS, L. y cols.: "Malignant perivascular epithelioid cell tumor (PEComa) arising in the broad ligament". Int. J. Gynecol. Cancer, 14: 1036, 2004.

5. FUKUNAGA, M.: "Perivascular epithelioid cell tumor of the uterus: A case report". Int. J. Gynecol. Pathol., 23: 287, 2004.

*6. BONETTI, F.; PEA, M.; MARTIGNONI, G. y cols.: "PEC and sugar". Am. J. Surg. Pathol., 16: 307, 1992.

*7. ZAMBONI, G.; PEA, M.; MARTIGNONI, G. y cols.: "Clear cell "sugar" tumor of the pancreas: A novel member of the family of lesions characterized by the presence of perivascular epitheliois cells". Am. J. Surg. Pathol., 20: 722, 1996.

*8. FOLPE, A.L.: "Neoplasms with perivascular epithelioid differenciation (PEComas)" In: Fletcher CDM, Unni KK, Merten LF (eds) Pathology and genetic of tumors of soft tissue and bone. Lyon. France. IARC Press, 2002.

**9. FOLPE, A.; MENTZEL, T.; LEER, H. y cols.: "Perivascular epithelioid cell neoplasms of soft tissue and gynecologic origin”. Am. J. Surg. Pathol., 29: 1558, 2005.

*10. WEINREB, I.; HOWART, D.; LATTA, E. y cols.: "Perivascular epithelioid cell neoplasms (PEComas): Four malignant cases expanding the histopathological spectrum and a description of a unique finding". Virchows Arch., 450: 463, 2007. 